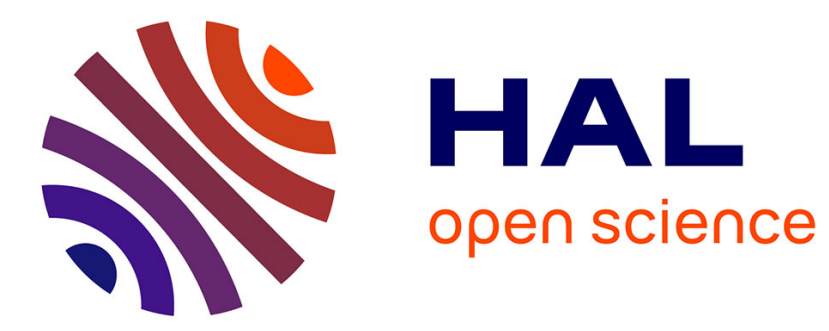

\title{
Charge transfer between atomic and ionic oxygen
}

\author{
Z. Pawlowska, Pierre Fauchais
}

\section{To cite this version:}

Z. Pawlowska, Pierre Fauchais. Charge transfer between atomic and ionic oxygen. Journal de Physique, 1984, 45 (5), pp.867-872. 10.1051/jphys:01984004505086700 . jpa-00209819

\section{HAL Id: jpa-00209819 https://hal.science/jpa-00209819}

Submitted on 1 Jan 1984

HAL is a multi-disciplinary open access archive for the deposit and dissemination of scientific research documents, whether they are published or not. The documents may come from teaching and research institutions in France or abroad, or from public or private research centers.
L'archive ouverte pluridisciplinaire HAL, est destinée au dépôt et à la diffusion de documents scientifiques de niveau recherche, publiés ou non, émanant des établissements d'enseignement et de recherche français ou étrangers, des laboratoires publics ou privés. 
Classification

Physics Abstracts

$31.20 \mathrm{~L}-34.70$

\title{
Charge transfer between atomic and ionic oxygen
}

\author{
Z. Pawlowska (*) and P. Fauchais \\ Equipe Thermodynamique et Plasma, LA 320, C.N.R.S., \\ Université de Limoges, 123, avenue Albert Thomas, 87060 Limoges Cedex, France
}

(Reçu le 2 mai 1983, révisé le 10 novembre, accepté le 11 janvier 1984)

\begin{abstract}
Résumé. - Nous avons calculé la section efficace de transfert de charge résonnant entre ion et atome d'oxygène. Les calculs ont été faits en utilisant la méthode du paramètre d'impact. Les coefficients des équations couplées ont été calculés sous forme analytique grâce à l'utilisation du développement des orbitales de Slater à deux centres de symétrie $p$ et du potentiel atomique de Green, Sellin et Zachor (GSZ).
\end{abstract}

\begin{abstract}
A calculation of a symmetric resonant charge exchange cross-section between atomic and ionic oxygen has been performed using the impact parameter method. The coefficients of the coupled equations are derived in an analytical form using a two centre expansion in the Slater orbitals of p-symmetry and the phenomenological atomic potential of Green, Sellin and Zachor (GSZ).
\end{abstract}

\section{Introduction.}

The resonant charge transfer cross-section has been calculated for a number of simple cases such as hydrogen and helium atoms but only a few papers have presented estimates of this cross-section for a more complex atom like oxygen (for review see [1-3]). In this case an extremely difficult experiment was performed by Stebbings $e t$ al. [4] using a cross-beam technique in the energy range $0.01-10 \mathrm{keV}$. The autors estimated the experimental error to be in the range $\pm 25 \%$, mainly due to the possible presence of excited ions in the primary beam. Dalgarno [5] used a semi-empirical method to predict the charge transfer cross-section on the basis of experimental for ion mobilities in their parent gases at low field strengths.

As is shown in section 2 (formulae (6)-(9)). The cross section of a symmetric process in the two stateapproximation depends only on the difference between the symmetric $E_{\mathrm{g}}$ and antisymmetric $E_{\mathrm{u}}$ stationary state energies, $\Delta E=E_{\mathrm{u}}-E_{\mathrm{g}}$, along the collision orbit.

Knof et al. [16] approximated this energy splitting for oxygen $\pi$ states (as obtained directly from the fitted empirical curves for $\mathrm{X}^{2} \pi_{\mathrm{g}}$ and $\mathrm{A}^{2} \pi_{\mathrm{u}}$ states) by a single exponential term (1) with two fitting parameters. These results were compared to the data obtained on the

(*) Permanent address : Institute for Low Temperature and Structure Research, Polish Academy of Sciences, pl. Katedralny 1, 50-329 Wroclaw, Poland. basis of the formulae of Rapp et al. [7], where the energy difference $\Delta E$ was also approximated by a single exponential, but with different parameters, which are in a rather rough correlation with the size of an electronic orbital through the ionization potential $I(2)$

$$
\begin{aligned}
& \Delta E=410 \exp (-3.489 R)[\mathrm{eV}], \quad R>1.95 \AA,(1) \\
& \Delta E=2 I R \exp (-\sqrt{I} R)[\mathrm{eV}]
\end{aligned}
$$

where $R$ is the internuclear distance.

The above assumption concerning the form of $\Delta E$ for atoms other than hydrogen, is coarse approximation, especially when the outer electron is not in an s-state.

The purpose of the present work is to present a new calculation using the impact parameter method, in which the total wave function has been expressed as a set of atomic orbitals having the proper p-symmetry. The analytical forms of the coefficients of the coupled equations have been derived and evaluated in terms of the phenomenological atomic potential GSZ $[9,10]$ and the single-zeta functions of Clementi and Roeti [8], see also [16]. These functions have the correct radial dependence at large internuclear separations, which is the region of importance for the cross-section calculation.

On the basis of this evaluations preliminary results concerning the probabilities and the cross-sections have been calculated in the two-state approximation. 


\section{Theory.}

The collision between an ion $\mathrm{O}^{+}$and its parent atom $\mathrm{O}$ may be described as

$$
\mathrm{O}_{\mathrm{A}}+\mathrm{O}_{\mathbf{B}}^{+} \rightarrow \mathrm{O}_{\mathrm{A}}^{+}+\mathrm{O}_{\mathbf{B}}
$$

where the nuclei $A$ and $B$ are assumed to be identifiable before and after collision. Within the impact parameter method it is assumed that the projectile moves along a rectilinear trajectory with a constant velocity $\bar{v}$ parallel to the fixed $\mathrm{z}$ axis (Fig. 1), so that the internuclear distance $2 . R$ is given by

$$
\text { 2. } R=\sqrt{b^{2}+v^{2} t^{2}},
$$

where $b$ is the impact parameter, and where the time $t$ varies from $-\infty$ to $+\infty$. The above assumption limits our approach to the intermediate relative velocity range $(100 \mathrm{eV} \div 10 \mathrm{keV})$; down to zero energy the assumption of the straight-line paths is not valid, while at high energies not only the separation of the electronic and atomic motions becomes difficult but also the impact parameter becomes so small that the couplings to all other electronic states must be taken into account. The atomic wave-functions : $u_{1}\left(\bar{r}_{\mathrm{A}, \mathrm{B}}\right)=u_{\mathrm{p}_{\mathbf{z}^{\prime}}}\left(\bar{r}_{\mathrm{A}, \mathrm{B}}\right), u_{2}\left(\bar{r}_{\mathrm{A}, \mathrm{B}}\right)=u_{\mathrm{p}_{x^{\prime} y^{\prime}}}\left(\bar{r}_{\mathrm{A}, \mathbf{B}}\right)$, and the potentials $V\left(\bar{r}_{A}\right)$ and $V\left(\bar{r}_{B}\right)$ are defined in the rotating coordinate system $\left(x^{\prime}, y^{\prime}, z^{\prime}\right)$ such that the $z^{\prime}$ axis coincides with $\bar{R}$ (Fig. 1).

The complete electronic wave-function of an active electron is written as a linear combination of traveling orbitals. Inserting this expansion into the Schrödinger equation leads to a set of coupled linear firstorder differential equations for the expansion coefficients $a_{i}$, which are functions of the time, depending parametrically on the impact parameter $b$ and the velocity $v$.

$$
A=\left|\begin{array}{cccc}
\frac{S_{1} J_{1}-I_{1}}{S_{1}^{2}-1} & -i \beta & \frac{S_{1} I_{1}-J_{1}}{S_{1}^{2}-1} & 0 \\
i \beta & \frac{S_{2} J_{2}-I_{2}}{S_{2}^{2}-1} & 0 & \frac{S_{2} I_{2}-J_{2}}{S_{2}^{2}-1} \\
\frac{S_{1} I_{1}-J_{1}}{S_{1}^{2}-1} & 0 & \frac{S_{1} J_{1}-I_{1}}{S_{1}^{2}-1} & -i \beta \\
0 & \frac{S_{2} I_{2}-J_{2}}{S_{2}^{2}-1} & i \beta & \frac{S_{2} J_{2}-I_{2}}{S_{2}^{2}-1}
\end{array}\right|
$$

where :

$$
\beta=\frac{b v}{4 R}
$$

and

$$
\begin{aligned}
& J_{1}=\left\langle u_{1}\left(\bar{r}_{\mathrm{A}}\right)\left|V\left(\bar{r}_{\mathrm{B}}\right)\right| u_{1}\left(\bar{r}_{\mathrm{B}}\right)\right\rangle \\
& I_{1}=\left\langle u_{1}\left(\bar{r}_{\mathrm{A}}\right)\left|V\left(\bar{r}_{\mathrm{B}}\right)\right| u_{1}\left(\bar{r}_{\mathrm{A}}\right)\right\rangle, \\
& S_{1}=\left\langle u_{1}\left(\bar{r}_{\mathrm{A}}\right) \mid u_{1}\left(\bar{r}_{\mathrm{B}}\right)\right\rangle .
\end{aligned}
$$

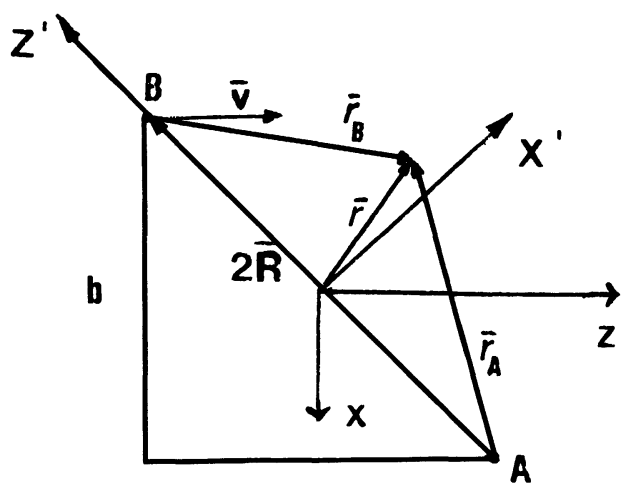

Fig. 1. - Coordinate systems used in the collision process : $\left(x^{\prime} y^{\prime} z^{\prime}\right)$ is the rotating coordinate system, $(x y z)$ is the fixed coordinate system, $R(t)$ is half the internuclear distance, $b$ is the impact parameter, A and B are the time dependent positions of the target (A) and the projectile (B) moving with constant velocity $\bar{v}$. The collision plane is chosen to be the $x z$ plane.

Neglecting, in the final analysis, the momentum of the active electron (we assume $\exp (-i \bar{v} z)=1$ ) the coupled equations for the charge exchange have the following form [11] :

$$
i \dot{a}=A a,
$$

here $a$ is the vector of transition amplitudes $\left(a_{1}=a_{1}^{\mathbf{A}}\right.$,

$$
a_{2}=a_{2}^{\mathrm{A}}, a_{3}=a_{1}^{\mathrm{B}}, a_{4}=a_{2}^{\mathrm{B}} \text { ) }
$$

$\dot{a}$ is the time derivative of $a$,

and the matrix of coefficients $A$ is determined by the operation of the time dependent Hamiltonian $H=-\frac{1}{2} \Delta+V\left(\bar{r}_{\mathrm{A}}\right)+V\left(\bar{r}_{\mathrm{B}}\right)$ on the total electron wave-function $\left({ }^{1}\right)$ :

The corresponding integrals with the subscript 2 are expressed by the functions $u_{2}$. It is well known that the diagonal elements of (4) may be removed by an appropriate choice of new transition amplitudes which

( ${ }^{1}$ ) Atomic units have been used, except where otherwise mentioned. 
only differ by a phase factor from the old ones. Then, in the two-state approximation, i.e. taking into account only $2 \mathrm{p}_{x^{\prime} y^{\prime}}^{A, B}$ wave functions (3) and (4) reduce to :

$$
\begin{aligned}
& \dot{i a}_{2}=A_{24} a_{4}, \\
& i \dot{a}_{4}=A_{42} a_{2},
\end{aligned}
$$

with $A_{24}=A_{42}=\left(S_{2} I_{2}-J_{2}\right) /\left(S_{2}^{2}-1\right)$. Uncoupling the two equations (6) by adding and subtracting them it may be seen that

$$
\left|a_{4}(\infty)\right|=\left|\sin \int_{-\infty}^{+\infty} A_{24} \mathrm{~d} t\right|,
$$

where $A_{24}$ corresponds to the difference between the symmetric and antisymmetric stationary states energies calculated within the molecular orbital linear combinations of atomic orbitals (MO LCAO) approximation for $\pi_{\mathrm{g}}$ and $\pi_{\mathrm{u}}$ states. Having solved (6) for $a_{2}, a_{4}$ and taking into account the initial condition: $a_{2}(-\infty)=1 ; a_{4}(-\infty)=0$, the charge transfer $\mathrm{A} \rightarrow \mathrm{B}$ probability is defined as

$$
P(b)=\left|a_{4}(t=+\infty)\right|^{2}
$$

and the corresponding charge transfer cross-section $Q$ is obtained by integrating $P$ over all impact parameters $b$ :

$$
Q=2 \pi \int_{0}^{\infty} b P(b) \mathrm{d} b
$$

\section{Results and discussion.}

As basis functions $u_{i}$ we have used $2 p$ oxygen single zeta functions [8] which are summarized in table I. A single zeta function is that approximation to the Hartree-Fock function, in which a given electron orbital is decribed by one Slater function. This function is more rudimentary than a double zeta function which is nearly as accurate as a Hartree-Fock function. In figure 2, we have compared both the above functions with the Hartree-Fock $2 \mathrm{p}$ wave function of the oxygen atom, which we have calculated self-consistently.

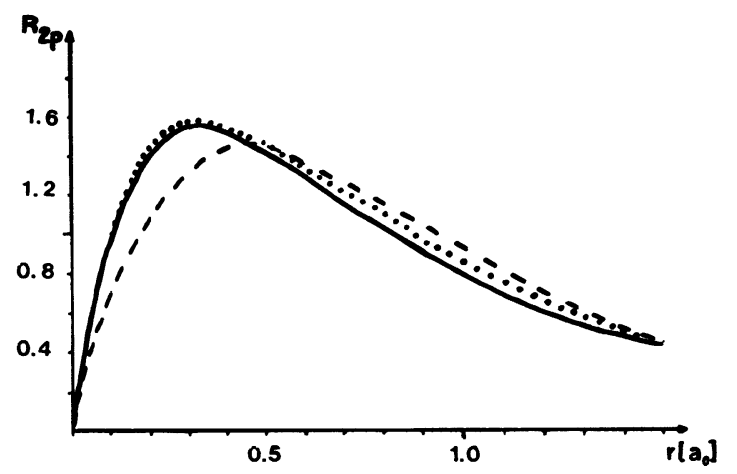

Fig. 2. - The radial parts of the oxygen $2 p$ wave functions :

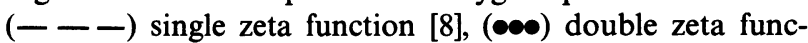
tion [8], ( $\longrightarrow$ Hartree-Fock self-consistent wave function.

Table I. - The atomic orbitals $(A O)$ of oxygen : single and double zeta functions [8] and the corresponding energies of the electronic level.

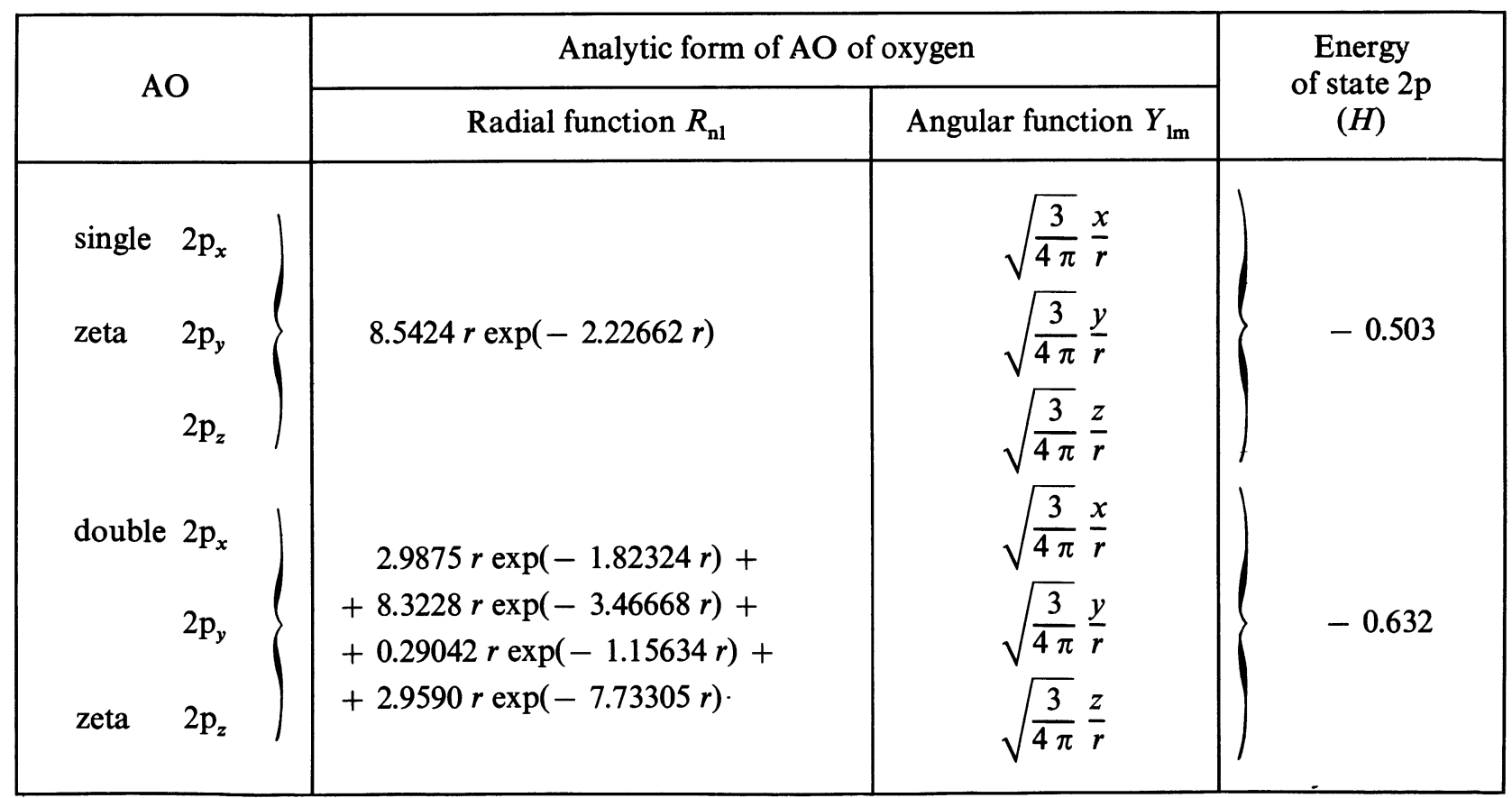


The GSZ form [9] of the atomic potential has been chosen :

$$
V\left(r_{\mathrm{A}, \mathrm{B}}\right)=\frac{-Z}{r_{\mathrm{A}, \mathbf{B}}}\left[(1-p) \omega\left(r_{\mathrm{A}, \mathrm{B}}\right)+p\right],
$$

in order to evaluate the elements of the matrix $A(4)$.

In the above formula $Z$ is the atomic number, $p=\frac{\theta+1}{Z}, \theta-$ is the degree of ionization and the screening function $\omega$ may be written

$$
\omega(r)=[K d(\exp (r / d)-1)+1]^{-1},
$$

$K$ and $d$ being parameters.

The GSZ atomic potential has a particularly useful parametric and analytic form, which has proven to be successful in computing atomic properties to a very high accuracy [15]. It is easily applied to atoms and also to ions. The parameters $K$ and $d$ were calculated for all elements with atomic number $Z \leqslant 10$ [10].

$K=2.41\left(a_{0}^{-1}\right)$ and $d=0.735\left(a_{0}\right)$ give the best fitting to the Hartree-Fock total energy for oxygen. The comparison of the self-consistent Hartree-Fock

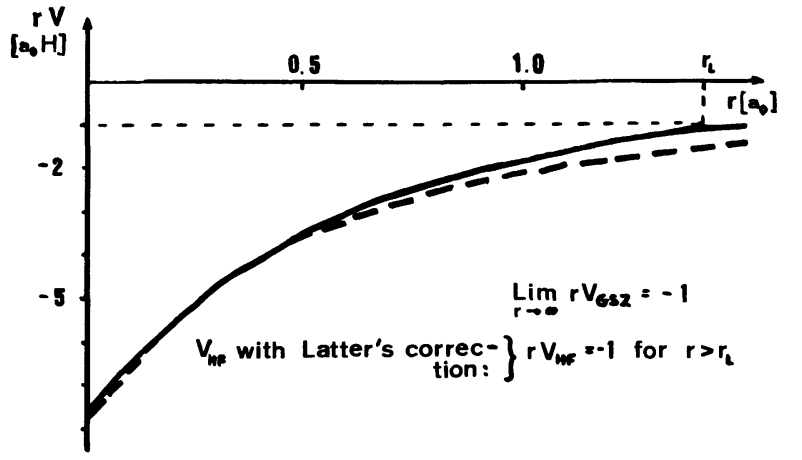

Fig. 3. - The dependence of $r . V(r)$ as a function of electronnucleus distance $r$ for : (- - GSZ atomic potential [9] (—) Hartree-Fock self-consistent potential.

(with Latter's correction [17]), and GSZ potentials, which are shown in figure 3 , indicates a very good agreement of both potentials.

Then the integrals (5) can be calculated analytically in the prolate spheroidal coordinate system [13] :

$$
\begin{aligned}
\left.J_{1}=\frac{N(Z}{K d}-1\right) & \sum_{\mu=1}^{\infty}\left\{( - 1 ) ^ { \mu - 1 } ( \frac { K d - 1 } { K d } ) ^ { \mu } \left\{\mathrm { e } ^ { - 2 a R } \left[\frac{1}{(2 R)^{3}}\left(-\frac{6 d^{4}}{s^{3} \mu^{4}}+\frac{6 d^{3}}{s^{4} \mu^{3}}\right)\right.\right.\right. \\
& +\frac{1}{(2 R)^{2}}\left(-\frac{12 d^{4}}{s^{3} \mu^{4}}+\frac{12 d^{3}}{s^{3} \mu^{3}}-\frac{3 d^{2}}{s^{4} \mu^{2}}\right)+\frac{1}{(2 R)}\left(-\frac{6 d^{4}}{s \mu^{4}}+\frac{9 d^{3}}{s^{2} \mu^{3}}-\frac{9 / 2 d^{2}}{s^{3} \mu^{2}}+\frac{3 / 8 d}{s^{4} \mu}\right) \\
& \left.+\left(\frac{4 d^{3}}{s \mu^{3}}-\frac{3 d^{2}}{s^{2} \mu^{2}}+\frac{d}{s^{3} \mu}\right)+(2 R)\left(-\frac{1 / 4 d^{2}}{s \mu^{2}}+\frac{1 / 8 d}{s^{2} \mu}\right)-(2 R)^{2}\left(\frac{3 / 2}{s \mu}\right)\right]+\exp \left[-2 R\left(a+\frac{\mu}{d}\right)\right] \\
& \times\left[\frac{1}{(2 R)^{3}}\left(\frac{12 d^{4}}{s^{3} \mu^{4}}-\frac{6 d^{3}}{s^{4} \mu^{3}}\right)+\frac{1}{(2 R)^{2}}\left(\frac{12 d^{4}}{s^{2} \mu^{4}}-\frac{3 d^{2}}{s^{4} \mu^{2}}\right)+\frac{1}{(2 R)}\left(\frac{6 d^{4}}{s \mu^{4}}+\frac{3 d^{3}}{s^{2} \mu^{3}}-\frac{3 / 2 d^{2}}{s^{3} \mu^{2}}-\frac{3 / 4 d}{s^{4} \mu}\right)\right. \\
+ & \left.\left.\left.\left(\frac{2 d^{3}}{s \mu^{3}}-\frac{1 / 2 d}{s^{3} \mu}\right)+(2 R)\left(-\frac{3 / 4 d^{2}}{s \mu^{2}}+\frac{3 / 8 d}{s^{2} \mu}\right)+(2 R)^{2}\left(\frac{d}{s \mu}\right)\right]\right\}\right\} \\
+ & N \mathrm{e}^{-2 a R}\left[\frac{1}{4 a^{4}}+\frac{(2 R)}{4 a^{3}}-\frac{3(2 R)^{2}}{8 a^{2}}-\frac{11(2 R)^{3}}{24 a}\right]
\end{aligned}
$$$$
I_{1}=\frac{N(Z-1)}{K d-1} \sum_{\mu=1}^{\infty}\left\{( - 1 ) ^ { \mu - 1 } ( \frac { K d - 1 } { K d } ) ^ { \mu } \left\{-\mathrm{e}^{-4 a R}\left[\frac{1}{(2 R)^{3}}\left(\frac{3 / 4}{s^{4} w^{3}}-\frac{3 / 4}{s^{3} w^{4}}\right)\right.\right.\right.
$$$$
+\frac{1}{(2 R)^{2}}\left(+\frac{3 / 4}{s^{4} w^{2}}-\frac{3 / 2}{s^{3} w^{3}}+\frac{3 / 4}{s^{2} w^{4}}\right)+\frac{1}{(2 R)}\left(\frac{3 / 8}{s w^{4}}-\frac{9 / 8}{s^{2} w^{3}}+\frac{9 / 8}{s^{3} w^{2}}-\frac{3 / 8}{s^{4} w}\right)+\left(\frac{1 / 2}{s w^{3}}+\frac{3 / 4}{s^{2} w^{2}}-\frac{1 / 2}{s^{3} w}\right)
$$$$
\left.+(2 R)\left(\frac{1 / 16}{s w^{2}}-\frac{5 / 16}{s^{2} w}\right)+R^{2}\left(\frac{3 / 8}{s w}\right)\right]+\exp \left(-2 R \frac{\mu}{d}\right)\left[\frac{1}{(2 R)^{3}}\left(\frac{3 / 4}{s^{4} w^{3}}-\frac{3 / 4}{s^{3} w^{4}}\right)\right.
$$$$
+\frac{1}{(2 R)^{2}}\left(\frac{3 / 4}{s^{2} w^{4}}-\frac{3 / 4}{s^{4} w^{2}}\right)+\frac{1}{(2 R)}\left(\frac{3 / 8}{s w^{4}}+\frac{3 / 8}{s^{2} w^{3}}-\frac{3 / 8}{s^{3} w^{2}}-\frac{3 / 8}{s^{4} w}\right)+\left(\frac{1 / 4}{s w^{3}}-\frac{1 / 4}{s^{3} w}\right)
$$$$
\left.\left.\left.+(2 R)\left(\frac{-3 / 16}{s w^{2}}-\frac{1 / 16}{s^{2} w}\right)+(2 R)^{2}\left(\frac{3 / 8}{s w}\right)\right]\right\}\right\}+N \mathrm{e}^{-4 a R}\left[\frac{1}{(2 R)^{3}}\left(\frac{-3 / 2}{(a)^{7}}\right) \frac{1}{(2 R)}\left(\frac{3}{a^{6}}\right)-\frac{1}{(2 R)}\left(\frac{3}{a^{5}}\right)\right.
$$$$
\left.\times\left(\frac{7}{4 a^{4}}\right)-\left(\frac{2 R}{8 a^{3}}\right)\right]+\left[\frac{1}{(2 R)^{3}}\left(\frac{3 / 2}{a^{7}}\right)-\left(\frac{3 R}{8 a^{8}}\right)\right]
$$ 


$$
\begin{aligned}
& J_{2}=\frac{N(Z-1)}{K d-1} \sum_{\mu=1}^{\infty}\left\{( - 1 ) ^ { \mu - 1 } ( \frac { K d - 1 } { K d } ) ^ { \mu } \left\{\mathrm { e } ^ { - 2 R a } \left[\frac{1}{(2 R)^{3}}\left(-\frac{6 d^{3}}{s^{4} \mu^{3}}+\frac{12 d^{4}}{s^{3} \mu^{4}}\right)\right.\right.\right. \\
& \left.+\frac{1}{(2 R)^{2}}\left(\frac{12 d^{4}}{s^{2} \mu^{4}}-\frac{12 d^{3}}{s^{3} \mu^{3}}+\frac{3 d^{2}}{s^{4} \mu^{2}}\right)+\frac{1}{(2 R)}\left(-\frac{8 d^{3}}{s^{2} \mu^{3}}+\frac{4 d^{2}}{s^{3} \mu^{2}}\right)+\frac{2 d^{2}}{s^{2} \mu^{2}}\right]+\exp \left[-2 R\left(a+\frac{\mu}{a}\right)\right] \\
& \left.\left.\times\left[\frac{1}{(2 R)^{3}}\left(\frac{6 d^{3}}{s^{4} \mu^{3}}-\frac{12 d^{4}}{s^{3} \mu^{4}}\right)+\frac{1}{(2 R)^{2}}\left(-\frac{12 d^{4}}{s^{2} \mu^{4}}+\frac{3 d^{2}}{s^{4} \mu^{2}}\right)+\frac{1}{(2 R)}\left(-\frac{4 d^{3}}{s^{2} \mu^{3}}+\frac{2 d^{2}}{s^{3} \mu^{2}}\right)\right]\right\}\right\} \\
& +N \mathrm{e}^{-2 a R}\left[\frac{1}{2 a^{4}}+\frac{(2 R)}{2 a^{3}}+\frac{(2 R)^{2}}{6 a^{2}}\right] \\
& I_{2}=\frac{N(Z-1)}{K d-1} \sum_{\mu=1}^{\infty}\left\{( - 1 ) ^ { \mu - 1 } ( \frac { K d - 1 } { K d } ) ^ { \mu } \left\{\mathrm { e } ^ { - 4 a R } \left[\frac{1}{(2 R)^{3}}\left(\frac{-3 / 4}{s^{4} w^{3}}+\frac{3 / 4}{s^{3} w^{4}}\right)+\right.\right.\right. \\
& \left.+\frac{1}{(2 R)^{2}}\left(\frac{3 / 4}{s^{2} w^{4}}-\frac{3 / 2}{s^{3} w^{3}}+\frac{3 / 4}{s^{4} w^{2}}\right)+\frac{1}{(2 R)}\left(\frac{-1}{s^{2} w^{3}}+\frac{1}{s^{3} w^{2}}\right)+\left(\frac{1 / 2}{s^{2} w^{2}}\right)\right] \\
& \left.\left.+\exp \left(-2 R \frac{\mu}{d}\right)\left[\frac{1}{(2 R)^{3}}\left(\frac{3 / 4}{s^{4} w^{3}}-\frac{3 / 4}{w^{4} s^{3}}\right)+\frac{1}{(2 R)^{2}}\left(\frac{-3 / 4}{s^{2} w^{4}}+\frac{3 / 4}{s^{4} w^{2}}\right)+\frac{1}{2 R}\left(\frac{-1 / 2}{s^{2} w^{3}}+\frac{1 / 2}{s^{3} w^{2}}\right)\right]\right\}\right\} \\
& +N \mathrm{e}^{-4 a R}\left[\frac{1}{(2 R)^{3}}\left(\frac{3}{2 a^{7}}\right)+\frac{1}{(2 R)^{2}}\left(\frac{3}{a^{6}}\right)+\frac{1}{(2 R)}\left(\frac{1}{a^{5}}\right)+\left(\frac{1}{2 a^{4}}\right)\right]+N\left[\frac{1}{(2 R)^{3}}\left(\frac{-3}{2 a^{7}}\right)+\frac{1}{(R \cdot 2)}\left(\frac{1}{a^{5}}\right)\right] \\
& S_{1}=N \mathrm{e}^{-2 a R}\left[\frac{1}{2 a^{5}}+\frac{(2 R)}{2 a^{4}}-\frac{3 / 10(2 R)^{2}}{a^{3}}-\frac{2 / 5(2 R)^{3}}{a^{2}}-\frac{5 / 32(2 R)^{4}}{a}\right] \\
& S_{2}=N \mathrm{e}^{-2 a R}\left[\frac{1}{a^{5}}+\frac{(2 R)}{a^{4}}+\frac{2 / 5(2 R)^{2}}{a^{3}}+\frac{1 / 15(2 R)^{3}}{a^{2}}\right]
\end{aligned}
$$

where $N=54.729(\mathrm{au})$ is the normalization constant, $a$ is an exponent of the atomic wave function (see Table I), $a=2.22662\left(a_{0}^{-1}\right)$, while $s=2 a+\mu / d$ and $w=-2 a+\mu / d$.

The last parenthesis of each expression (12-15) corresponds to the part of the atomic potential (10) $V(r) \sim-1 / r$, which is equivalent to the hydrogen atom potential. Therefore, this part of our results corresponds to the results of Rapp and Dinwiddie [14] for $2 \mathrm{p}_{1}$ hydrogen atom states.

The dependences of $J_{\mathbf{p}_{x y}}, I_{\mathbf{p}_{x y}}$ and $S_{\mathbf{p}_{x y}}$ as on the internuclear distance are presented in figure 4.

It is evident from our calculations that even in the most simple case when we assume $S_{2}=0$, in the whole range of internuclear distances at least two exponential terms are necessary to describe $A_{24}=J_{2}$. The first one (for $\mu=1$ ) describes the screening of the nucleus, particularly important for short and middle internuclear distances. The second exponential function describes the oxygen atom in terms of the hydrogen-like model, for large distances. In previous publications $[6,7]$, this difference between symmetric and antisymmetric stationary states energies was approximated by a single exponential term (1), (2).

All the above results (12-17) can be easily transformed if the double zeta function is used instead of the single zeta function. In this case the magnitudes $J, I$ and $S$ can be expressed as linear combinations of terms corresponding to the single zeta function, in which 2a should be replaced by a sum of two different

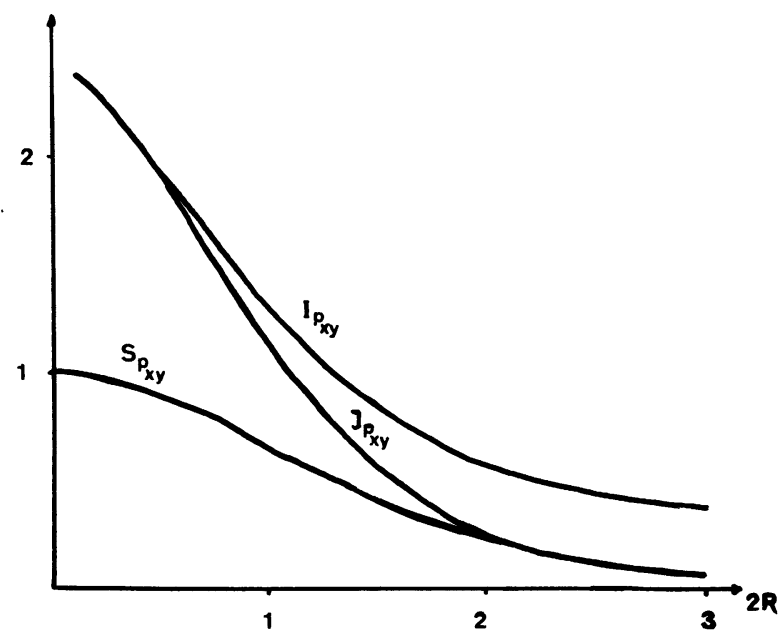

Fig. 4. $-J_{2}, I_{2}$ and $S_{2}$ dependence on the internuclear distance $2 R$. For further explications see the text.

(in general) exponents $\left(a_{1}+a_{2}\right)$ and $N$ by the product of the coefficients in front of the exponentials (see Table I).

We have solved (3) in the two-state approximation using Hamming's predictor-corrector method [12]. Our results for the cross-sections as a function of the oxygen energy in the range $0.100-10 \mathrm{keV}$ are presented in figure 5 as compared to the experimental data [4], and the previous theoretical results $[6,7]$. 


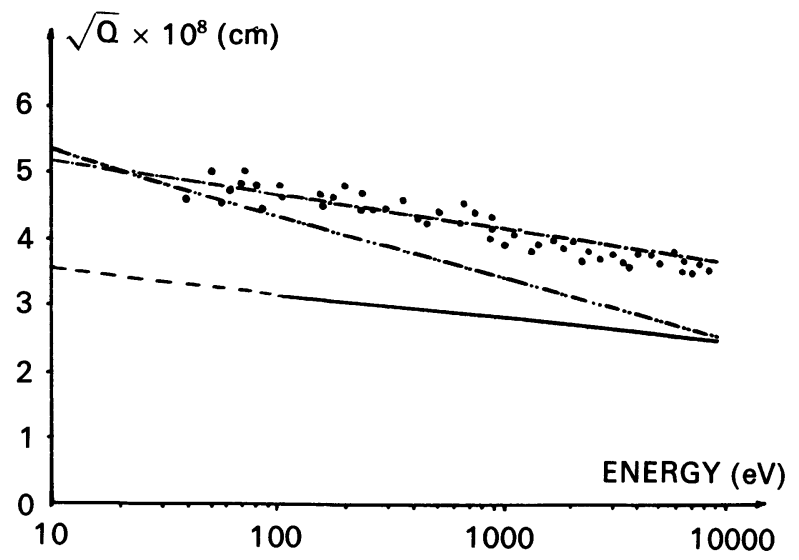

Fig. 5. - The comparison of the experimental data of Stebbings [4] denoted by points with the semi-empirical results of Rapp et al. [14] ( $\longrightarrow \infty)$ ) and Knof et al. [6] ( $\longrightarrow-)$ and our theoretical results (-). Below the range $100 \mathrm{eV} \div 10 \mathrm{keV}$, the theoretical results are denoted by a dashed curve.

The calculated data differ slightly from the experimental results. There are several reasons for this. First, our results based on the two-state approximation neglect all nonadiabatic transitions to higher $\mathrm{O}_{2}^{+}$ states, whereas the actual experiment may probably involve charge exchange process with excitation. Furthermore, the initial ion beam used in experiment [4] contained excited states of $\mathrm{O}^{+}$which could react analogously to the ground state of $\mathrm{O}^{+}$, but involving other states. Finally in the two-state approximation the rotational coupling terms were neglected as are, obviously all dynamic interactions.

\section{Conclusions.}

Contrary to approaches $[6,7]$ which were fitted to the experimental data, we have presented a fully theoretical model to calculate the charge exchange crosssections for the p-electron atoms, in the intermediate energy region, where the semiclassical impact parameter method is applicable.

The total wave function of an active electron has been expressed as a set of atomic orbitals having a proper p-symmetry. Then the analytical forms of the coefficients of the coupled equations have been derived and evaluated in terms of a single zeta function and of the phenomenological atomic GSZ potential. These equations have been numerically solved using Hamming's predictor-corrector method for the transition amplitudes. The charge exchange cross-section for the oxygen resonant process which was calculated on the basis of these amplitudes shows a satisfactory agreement with the experimental data.

In order to obtain a better accuracy our model can be easily extended to take into account the double zeta function (see Fig. 2) and the additional states of s-symmetry. This more sophisticated and more time consuming extension of the calculations above is in progress.

\section{References}

[1] Basu, D., Mukherjee, S. C., Sural A., Phys. Reports 56 (1979) 279.

[2] Nikitin E. E., Theory of Elementary Atomic and Molecular Processes in Gases (Clarendon, Oxford) 1974.

[3] Delos, J. B., Rev. Mod. Phys. 53 (1981) 287-357.

[4] Stebings, R. F., Smith, A. C. H. and Ehrhardt, H., Proc. Third Int. Conf. on Phys. of Elec. and At. Collisions, London, 1963, ed. McDowell M.R.C. (North Holland, Amsterdam) 1964, p. 814-822.

[5] Dalgarno, A., Philos. Trans. Roy. Soc. A 250 (1958) 426-38.

[6] Knof, H., Mason, E. A. and Vanderslice, J. T., $J$. Chem. Phys. 40 (1964) 3548-53.

[7] Rapp, D. and Francis, W. E., J. Chem. Phys. 37 (1962) 2631-45.

[8] Clementi, E. and Roeti, C., Atomic Data and Nuclear Data Tables 14 (1974) 177-478.

[9] Green, A. E. S., Sellin, D. L. and Zachor, A. S., Phys. Rev. 184 (1969) 1-11.
[10] Szydlik, P. P. and Green, A. E. S., Phys. Rev. A 9 (1974) 1885-94.

[11] Van den Bos, J., J. Chem. Phys. 59 (1973) 5358-63.

[12] Van den Bos, J., Comp. Phys. Commun. 7 (1974) 163171.

[13] Ed. Abramowitz, M. and Stegun, I. A., Handbook of Mathematical Functions (Dover Public. Inc., New York) 1972.

There are errors in the formulae 21.5.1 and 21.5.2: instead of : $c=\frac{1}{2} f k$ should be $: c=f k$.

[14] RaP'P, A. and Dinwiddie, D., J. Chem. Phys. 57 (1972) 4919-27.

[15] Miller, K. J. and Green, A. E. S., J. Chem. Phys. 60 (1974) 2617-26.

[16] Hird, B. and Ali, S. P., Canad. J. Phys. 59 (1981) 576-584.

[17] LATter, R., Phys. Rev. 99 (1955) 510. 